\title{
LE RÔLE DES TOPOÏ DANS LA NÉGOCIATION CONVERSATIONNELLE ${ }^{1}$
}

\section{Les topoï: définition}

Les topoï apparaissent pour la première fois chez Aristote dans ses Topiques. Là, il étudie des espèces des topoï, pouvant servir de prémisses à des syllogismes dialectiques ou rhétoriques. Dans sa Rhétorique, il distingue les lieux communs, qui peuvent servir indifféremment en n'importe quelle science et ne relèvent d'aucune, et les lieux spécifiques, qui sont propres soit à une science particulière, soit à un genre oratoire bien défini (d'après Perelman et Olbrechts-Tytéca: 1958: 12).

Le "champ topique" est donc constitué d'une liste de thèmes, d'arguments généraux, dont l'apprentissage est un des objets essentiels de l'éducation rhétorique ancienne (Plantin 1990). Les topoï ou les lieux communs sont les reservoirs d'arguments, où l'on puise selon les besoins. Partant de la matière qui lui est fournie par la cause qu'il doit défendre, l'orateur doit mettre en oeuvre tous les arguments capables d'influencer au mieux le juge dans le sens des intérêts de la partie qu'il représente (Plantin, 1990: 237).

Les topoï chez Anscombre et Ducrot sont étymologiquement liés à Aristote, mais appartiennent conceptuellement à la théorie de l'argumentation dans la langue (Anscombre et Ducrot, 1983), d'après laquelle "les relations argumentatives ne sont pas surajoutées à la valeur sémantique de l'énoncé", mais sont "fondamentales, (...), présentes dès le niveau le plus profond de l'analyse" (Anscombre et al., 1995: 15).

D'après Anscombre (1995: 38-39), lors d'une énonciation, le locuteur donne des indications sur le chemin qu'il a choisi, et celui qui interprète tente de reconstruire un itinéraire à partir des indications fournies. Ces indications qui permettent d'opérer un choix parmi les chemins sont les topoï. Ce sont les principes généraux qui servent d'appui au raisonnement, mais ne sont pas le raisonnement. Ils ne sont jamais assertés, en ce sens que leur locuteur ne se présente jamais comme en étant l'auteur (même s'il l'est effectivement), mais ils sont utilisés. Ils sont toujours présentés comme faisant objet d'un consensus au sein d'une communauté plus ou moins vaste (y compris réduite à un individu, par exemple le locuteur).

1 Cette communication a été présentée en tant qu'affiche au cours du $6^{\text {ième }}$ Colloque international de la pragmatique à Reims du 19 au 24 juillet 1998. 
Le topos spécifie les prémisses qu'il est pertinent d'employer dans une situation et garantit le passage des prémisses vers la conclusion: il a donc la valeur du "garant" qui relève de sa nature procédurale. C'est pourquoi ils peuvent très bien être créés de toutes pièces, tout en étant présentés comme ayant force de loi, comme allant de soi (Ducrot, 1983).

Le topos est graduel: il met en relation deux prédicats graduels, deux "échelles", et la relation entre les deux échelles, elle aussi, est graduelle" (Ducrot 1995), comme le montre le schéma (1).

(1) 1 plus (moins) O est $P, \mp$ plus (moins) O' est P'

Ce schéma peut être illustré par l'exemple (2):

(2)

JS: Et ... de quoi parlons-nous? On parle de plus de 50000 d'entreprises de production, on parle de 20 milliards de chiffres d'affaires au niveau de la production et on parle de 35 légumes. Alors, aujourd'hui, aujourd'hui depuis que nous posons les questions au ministre le 25 juillet, le 25 novembre, le 6 avril et puis des séries de rencontres, on nous répond au niveau du Ministère, tout à coup on nous répond "choux-fleurs: Bretagne". Je rappelle: il y a 35 légumes, il y a une trentaine de départements concernés.

(Polémiques ${ }^{2}$ )

Le locuteur expose le topos T1: plus il y a de problèmes, plus il faut en parler. $\mathrm{Ce}$ topos est graduel: on parle beaucoup de choux-fleurs et de Bretagne. Mais il s'agit de 35 légumes et d'une trentaine de départements: il faut donc en parler davantage et trouver une solution plus complexe.

T1 met en relation deux échelles:

(3)

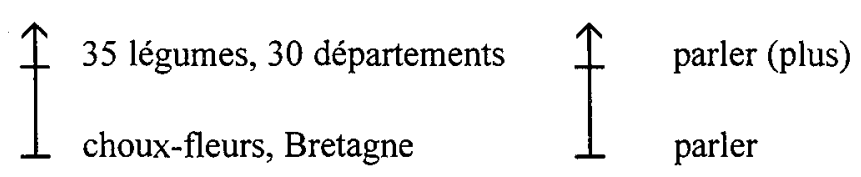

2 Cet extrait, aussi bien que les autres, cités au cours de la communication, appartient au corpus oral, enregistré et transcrit par M. Schlamberger Brezar. Les conventions de transcription sont les suivantes:

Majuscule: début de l'énoncé

$\mathrm{XXX}$ mot incompréhensible

$/ /$ intonation descendante

$/, /$ intonation montante

/... énoncé non achevé

(...) omission d'une partie du texte 


\section{Inventaire des topoï dans les textes analysés}

Nous allons essayer d'éclairer le rôle que jouent les topoï dans la négociation conversationnelle. ${ }^{3} \mathrm{Il}$ semble que, d'une part, l'orientation des topoï, leur pertinence et leur gradualité influencent le choix des moyens d'argumentation (les opérateurs et les connecteurs) et par conséquent de différentes relations syntaxiques sous-jacentes, et d'autre part elles programment l'issue de la négociation. En vue de démontrer cette hypothèse, nous avons analysé plusieurs simulations de négociations commerciales en langue slovène et un débat en français. ${ }^{4}$

D'après Plantin (1997: 12), le genre argumentatif se différencie en plusieurs espèces: certaines sont orientées vers l'expression et amplification, à savoir les débats d'idées, les débats politiques, d'autres vers la résolution du différend (négociations, conciliations). Nous avons donc affaire à deux types de textes différents où, nous supposons, les locuteurs vont faire preuve d'une attitude différente envers les topoï.

\subsection{Où placer les topoü dans la grammaire}

Les topoï ne sont pas assertés, ils sont sous-jacents et sous-entendus, posés comme garant. Dans la grammaire fonctionnelle (Dik, 1989) ils sont codés au niveau sous-jacent de la prédication, de l'état de choses. Ils représentent une connexion inférentielle entre les événements ou les propriétés par lesquelles les arguments sont liés à la conclusion (Ellerup-Nielsen, 1996: 330). La base de l'analyse des textes argumentatifs est l'énoncé, le niveau de "clause" (Dik, 1989). Le topos se trouve dans la structure sous-jacente de chaque énoncé argumentatif, servant de premisse pour une conclusion. C'est une entité sémantique.

Nolke (1994: 164) dit que

«l'argumentation effectivement accomplie n'est jamais précisée dans la signification, mais la langue pose sur l'acte de l'argumentation des contraintes sémantiques qui, elles, doivent être décrites dans la signification. Toute phrase isolée est susceptible d'entrer dans une vatiété apparemment illimitée de chaînes argumentatives,

3 La notion de la conversation comme négociation (Roulet et al., 1985: 9-11, Roulet, 1985: 13) suppose que dans une converstation, tout se négocie constamment: les modalités d'ouverture et de clôture de l'interaction. Il y a aussi l'idée que l'échange ne peut pas se conclure sans accord commun.

4 Nous avons analysé un débat télévisé, Les polémiques, diffusé sur la chaîne France 2, et 4 négociations commerciales en langue slovène. Les textes analysés appartiennent à deux genres différents de textes argumentatifs, les négociations étant orientées vers la résolution du différend et les débats vers l'expression et l'amplification publiques du différend (Plantin, 1997: 12). Or, les contraintes de conclusion s'imposent pour les deux genres. 
donnant lieu ainsi à une gamme non restreinte des topoï. Mais, d'une part, les expressions référentielles apportent avec elles tout un réseau de topoï relevant d'un champ topique, et, d'autre part, certains lexèmes - à savoir les opérateurs argumentatifs orientent ou dirigent le choix à l'intérieur de l'éventail de topoï ainsi présenté.»

La fonction du topos est d'une part de permettrte l'accès aux conclusions visées par l'acte d'argumentation, et, d'autre part, de choisir parmi les conclusions lorsque plus d'un acte d'argumentation est réalisé (Moeschler, 1985: 72). Le topos est de ce fait en étroit rapport avec les marques argumentatives que sont les opérateurs et les connecteurs, mais également avec la notion primitive d'acte d'orientation argumentative, puisque c'est à partir de telles indications que s'applique le topos. Il faut distinguer les connecteurs - prédicats à deux places et les connecteurs - prédicats à trois places (Moeschler, 1985). Les premiers relient deux échelles, les seconds deux topoï.

\subsection{Les topoï dans le débat}

"L'argumentation repose sur l'utilisation systématique de principes d'enchaînements d'énoncés ou "topoi" dont la validité est admise par des interlocuteurs" (Plantin, 1990: 246). Les topoï exposés au cours du débat ${ }^{5}$ télévisé peuvent être rassemblés dans les groupes suivants:

1. Topos "économique"

Plus on produit; plus on doit vendre.

Plus on a de produits, plus le prix est bas.

Plus le prix est bas, moins il faut produire.

\section{Topos "social"}

Plus on manque de ressources, plus on est obligé de descendre dans la rue.

Plus on est en crise, plus il faut faire appel à la collectivité.

Plus on est en crise, plus vite il faut agir.

Plus haut on est placé (à l'échelle sociale), plus on doit être au courant.

\section{Topos "violence"}

Meilleures raisons qu'on a, plus on est autorisé à descendre dans la rue.

Plus on est violent, moins c'est permis.

5 Dans le débat qui porte sur la violence des protestations des agriculteurs bretons et des agriculteurs en France en général, il y a les participants: MC; modératrice; GD, producteur, JS, FNPL (Fédération nationale des producteurs de légumes), FC, directeur du cabinet du Ministre de l'agriculture, MHA, députée des verts, CJ, député, $\mathrm{GG}$ et $\mathrm{EP}$, députés européens, JL, maire de Morlaix. 
Plus on est en crise, moins il faut être violent.

Plus il y a de dégats, plus il faut appliquer la loi.

4. Topos "qualité"

Plus on produit, plus la qualité baisse.

Plus il y a de qualité, plus ça coûte.

\section{Topos "débat"}

Plus il y a de problèmes, plus il faut en parler.

6. Topos "politique agricole commune"

Plus près on est de l'Euro, plus se règlent les aspects monétaires.

Plus le système est figé, plus il faut qu'il évolue.

Meilleur soit l'état de l'agriculture, plus il y a d'emplois.

Les topoï exposés de 1 à 6 sont pertinents pour le débat: l'argumentation se fait par la reprise ou par la réfutation du topos: c'est le "fil rouge" qui assure la suite du débat. C'est à l'aide du topos que s'enchaînent les arguments.

Illustration à l'aide d'un exemple du débat:

MHA: Je crois qu'effectivement aujourd'hui on est dans un système de politique agricole commune dont les aides favorisent cette surproduction. Or nos concitoyens, je crois que nous tous, nous attendons plutôt des produits de qualité. Donc... donc nous pensons qu'il faut la réformer profondément (la: politique agricole commune).

$\mathrm{T} 1$ : Plus on favorise la surproduction, plus on va produire.

T2: Plus on produit, moins les produits sont de qualité.

T3: Plus le consommateur est conscient, plus il exige des produits de qualité.

T4: Plus une politique est mauvaise, plus il faut la réformer.

Les topoï exposés sont relatifs à des idéologies banales de la vie quotidienne quant à l'usage qu'il convient de faire de son temps. Le premier énoncé comporte deux topoï sous-jacents, $\mathrm{T} 1$ et T2, dont le premier est mentionné et l'autre sousentendu. Aux deux s'oppose T3, introduit par le connecteur or, qui met la qualité en premier lieu. C'est sur ce point qu'est fondée la conclusion, introduite par le connecteur donc, qui appelle à la réforme.

La réponse de JS (2) s'enchaîne sur le T1: 
(5)

JS: Je ne peux pas rentrer dans votre sens. Aujourd'hui la production française satisfait à peine à autour de $70 \%$ de la consommation française. En Europe, la production des fruits ou des légumes et des fruits sont liés à la même problématique pour voir: Europe a à peine $40 \%$. L'Europe est le premier importateur mondial des fruits et des légumes. Donc je veux dire dans ces chiffres là qu'il faut intégrer, qu'il n'y a pas de surproduction. Par contre, il y a des téléscopages effectivement, et le cas des choux-fleurs est un téléscopage.

Dans sa réponse, JS déclare que la première échelle n'est pas pertinente, parce que on ne produit pas trop. Le rejet est fait d'une manière explicite ("Je ne peux pas rentrer dans votre sens") et démontré par le syllogisme:

Si l'on produit moins de $100 \%$ de sa consommation, il n'y a pas de surproduction. La France satisfait à $70 \%$ sa consommation de légumes.

(L'Europe satisfait à $40 \%$ sa consommation de légumes.)

Donc: il n'y a pas de surproduction.

Une autre réplique s'enchaîne sur le T3 d'abord et puis sur le T2.

(6)

EP: Je vais aussi signaler à Madame A.: des produits de qualité. Mais tous les agriculteurs ne souhaitent que ça de faire des produits de qualité. Mais les produits de qualité ont un coût. Et est-ce qu'on peut bien se poser la question si les consommateurs veulent bien payer ce coût. Or aujourd'hui nous nous apercevons que le pouvoir d'achat n'augmente que leur pouvoir d'achat ne leur permet pas... leur pouvoir d'achat ne leur permet pas de consommer cher.

EP: Tel que XXX il faut s'entendre qu'il y a à partir du collectivisme surproduction et que les produits ne sont pas de qualité. Je pense que c'est une idée reçue qui ne correspond pas à la réalité. Je ne peux pas XXX ressentir cela. La qualité aujourd'hui c'est une qualité qui est en France reconnue y compris à l'étranger et qui est tout à fait convenable. Il y a encore des progrès à faire et ils se font tous les jours. Mais on ne peut pas laisser dire cela. Donc...

Cette réplique s'enchaîne d'abord sur T3 et ajoute le nouveau topos T5: plus les produits sont de qualité, plus cela coûte. Mais d'autre part, il reconnaît le topos de "qualité", qui est reconnue en France et rejette le T2, qui lie la surproduction au manque de la qualité. 